Supplementary information

\title{
Characterization of the Key Aroma Compounds in a Commercial Milk Chocolate by Application of the
} Sensomics Approach

Kristina Fricke ${ }^{a}$ and Peter Schieberle ${ }^{b}{ }^{*}$

aLeibniz-Institute for Food Systemsbiology at the Technical University München (LSB@TUM; formerly Deutsche Forschungsanstalt für Lebensmittelchemie),

Lise-Meitner-Strasse 34, D-85354 Freising, Germany

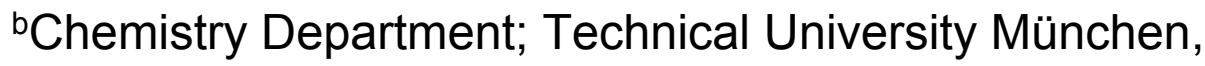
Lichtenbergstasse 4; 85478 Garching

${ }^{\star}$ Corresponding author:

E-mail: peter.schieberle@ch.tum.de

Phone 004987197698160 
Table S1 References for the synthesis of reference compounds and isotopically labeled internal standards.

\begin{tabular}{|c|c|}
\hline compound & literature \\
\hline 2-acetyl-1-pyrroline & $\begin{array}{l}\text { S1. Schieberle, P., Primary odorants in popcorn. J. Agric. Food Chem. 1991, 39, } 1141- \\
1144 .\end{array}$ \\
\hline (Z)-2-nonenal & $\begin{array}{l}\text { S2. Ullrich, F., Grosch, W., Flavour deterioration of soya-bean oil: Identification of } \\
\text { intense odour compounds formed during flavour reversion. Fat Sci Technol., 1988, 90, } \\
\text { 332-336. }\end{array}$ \\
\hline trans-4,5-epoxy-(E)-2-decenal & $\begin{array}{l}\text { S3. Schieberle, P., Grosch, W., Potent odorants of the wheat bread crumb. } \\
\text { Differences from the crust and effect of a longer dough fermentation. } Z \text {. } \\
\text { Lebensm. Unters. Forsch. 1991, 192, 130-135. }\end{array}$ \\
\hline$\left[{ }^{2} \mathrm{H}_{2}\right]$-decanoic acid & $\begin{array}{l}\text { S5. Guth, H., Quantitation and sensory studies of character impact odorants of } \\
\text { different white wine varieties. J. Agric. Food Chem. 1997, 45, 3027-3032. }\end{array}$ \\
\hline $\begin{array}{l}{\left[{ }^{13} \mathrm{C}_{2}\right]-\gamma \text {-decalactone }} \\
{\left[{ }^{2} \mathrm{H}_{2-4}\right]-\delta \text {-decalactone }} \\
{\left[{ }^{13} \mathrm{C}_{2}\right]-\gamma \text {-dodecalactone }} \\
{\left[{ }^{2} \mathrm{H}_{2-4}\right]-\delta \text {-dodecalactone }} \\
{\left[{ }^{13} \mathrm{C}_{2}\right]-\gamma \text {-nonalactone }} \\
{\left[{ }^{13} \mathrm{C}_{2}\right]-\gamma \text {-octactone }} \\
{\left[{ }^{2} \mathrm{H}_{2-4}\right]-\delta \text {-octalactone }} \\
{\left[{ }^{13} \mathrm{C}_{2}\right]-\gamma \text {-undecalactone }}\end{array}$ & $\begin{array}{l}\text { S6. Schütt, J., Schieberle, P. Quantitation of nine lactones in dairy cream by stable } \\
\text { isotope dilution assays based on novel syntheses of carbon- } 13 \text { labeled gamma } \\
\text { lactones and deuterium labeled delta-lactones in combination with comprehensive } \\
\text { two-dimensional gas chromatography with time-of-flight mass spectrometry. J. Agric. } \\
\text { Food Chem.,2017, 65,10534-10541. }\end{array}$ \\
\hline $\begin{array}{l}{\left[{ }^{2} \mathrm{H}_{3}\right]-2,3 \text {-diethyl-5-methylpyrazine }} \\
{\left[{ }^{2} \mathrm{H}_{3}\right] \text {-2-methoxyphenol }}\end{array}$ & $\begin{array}{l}\text { S7. Cerny, C.; Grosch, W., Quantification of character-impact odour compounds of } \\
\text { roasted beef. Eur. Food Res. Technol. 1993, 196, 417-422. }\end{array}$ \\
\hline$\left[{ }^{2} \mathrm{H}_{6}\right]$-dimethyl trisulfide & $\begin{array}{l}\text { S8. Milo, C., Aromastoffe von gekochter Forelle, gekochtem Kabeljau und Lachs vor } \\
\text { und nach Tiefkühllagerung des Rohmaterials. PhD thesis, Technical University } \\
\text { Munich, Germany, } 1995 \text {. }\end{array}$ \\
\hline
\end{tabular}




\begin{tabular}{|c|c|}
\hline & \\
\hline $\begin{array}{l}{\left[{ }^{13} \mathrm{C}_{2}\right]-4-h y d r o x y-2,5-\operatorname{dimethyl}-3(2 \mathrm{H})-} \\
\text { furanone }\end{array}$ & $\begin{array}{l}\text { S9. Blank, I.; Fay, L. B.; Lakner, F. J.; Schlosser, M., Determination of 4-Hydroxy-2,5- } \\
\text { dimethyl-3(2H)-furanone and } 2 \text { (or 5)-ethyl-4-hydroxy-5 (or } 2-\text {-)methyl-3 ( } 2 \text { H)-furanone } \\
\text { in pentose sugar-based Maillard model systems by isotope dilution assays. J. Agric. } \\
\text { Food Chem. 1997, 45, 2642-2648. }\end{array}$ \\
\hline$\left[{ }^{2} \mathrm{H}_{3}\right]$-3-methoxy-4-hydroxybenzaldehyde & $\begin{array}{l}\text { S10. Guth, H.; Grosch, W., Odorants of extrusion products of oat meal - changes } \\
\text { during storage. Eur. Food Res. Technol. 1993b, 196, 22-28. }\end{array}$ \\
\hline$\left[{ }^{2} \mathrm{H}_{3}\right]$-methanethiol & $\begin{array}{l}\text { S11. Guth, H.; Grosch, W., Identification of the character impact odorants of stewed } \\
\text { beef juice by instrumental analyses and sensory studies. J. Agric. Food Chem. 1994, } \\
42,2862-2866 \text {. }\end{array}$ \\
\hline$\left[{ }^{2} \mathrm{H}_{5-8}\right]-3-$ methylindole & $\begin{array}{l}\text { S12. Steinhaus, M.; Schieberle, P., Role of the fermentation process in off-odorant } \\
\text { formation in white pepper: On-site trial in Thailand. J.Agric. Food. Chem. 2005, } 53 \text {, } \\
6056-6050 .\end{array}$ \\
\hline $\begin{array}{l}{\left[{ }^{2} \mathrm{H}_{2}\right] \text {-methylpropanal }} \\
{\left[{ }^{2} \mathrm{H}_{2}\right]-2 \text {-methylbutanal }}\end{array}$ & $\begin{array}{l}\text { S13. Schieberle, P., Grosch, W., Changes in the concentrations of potent crust } \\
\text { odorants during storage of white bread. Flav. Fragr. J. 1992, } 7,213-218 \text {. }\end{array}$ \\
\hline$\left[{ }^{2} \mathrm{H}_{2}\right]-(E)-2-$ nonenal & $\begin{array}{l}\text { S14. Guth, H.; Grosch, W., Comparison of stored soy-bean oil and rapeseed oils by } \\
\text { aroma extract dilution analysis. Lebensm.-Wiss. U.-Technol. 1990, 23, 59-65. }\end{array}$ \\
\hline$\left[{ }^{2} \mathrm{H}_{5}\right]-2$-phenylethyl acetate & $\begin{array}{l}\text { S17. Schütt, J. On the role of lactones in the overall aroma of milk chocolate and their } \\
\text { formation during processing. } 2016, \mathrm{PhD} \text { thesis, Technical University of Munich. }\end{array}$ \\
\hline$\left[{ }^{13} \mathrm{C}_{2}\right]$-ethyl phenylacetate & $\begin{array}{l}\text { S18. The compound was synthesized by reacting }\left[{ }^{13} \mathrm{C}_{2}\right] \text {-phenylacetic acid with ethanol } \\
\text { (unlabeled: } 165 \mathrm{~m} / \mathrm{z} \text {, labeled: } 167 \mathrm{~m} / \mathrm{z} \text { ). }\end{array}$ \\
\hline
\end{tabular}

